\title{
Die Genveränderung von Embryonen bürdet Kindern eine unzumutbare Last auf
}

Hille Haker

Loyola University Chicago, hhaker@luc.edu

Follow this and additional works at: https://ecommons.luc.edu/theology_facpubs

Part of the Medical Humanities Commons, and the Religious Thought, Theology and Philosophy of Religion Commons

\section{Recommended Citation}

Haker, Hille. Die Genveränderung von Embryonen bürdet Kindern eine unzumutbare Last auf. Bioethica Forum, 10, 2: 50-59, 2017. Retrieved from Loyola eCommons, Theology: Faculty Publications and Other Works,

This Article is brought to you for free and open access by the Faculty Publications and Other Works by Department at Loyola eCommons. It has been accepted for inclusion in Theology: Faculty Publications and Other Works by an authorized administrator of Loyola eCommons. For more information, please contact ecommons@luc.edu. cc) (i) $\ominus$

This work is licensed under a Creative Commons Attribution-Noncommercial-No Derivative Works 3.0 License. (C) Swiss Society for Biomedical Ethics 2017 


\title{
Die Genveränderung von Embryonen bürdet Kindern eine unzumutbare Last auf
}

\author{
Hille Hakera \\ a Loyola University Chicago, USA
}

\section{Zusammenfassung}

Die Forschung an menschlichen Embryonen unterliegt in den meisten Staaten einer besonderen Kontrolle, die auch in den bestehenden internationalen Rechtsinstrumenten festgelegt ist. Angesichts der nun möglichen Keimbahnveränderungen durch das sogenannte «Gene Editing» oder «Genome Editing» ist eine öffentliche Diskussion aber nicht nur dringend notwendig, sondern überfällig. Die Wissenschaftsethik muss dabei eine grössere Rolle spielen, als dies derzeit der Fall ist. Meine Überlegungen in diesem Artikel betreffen ausschliesslich die reproduktive Anwendung, die ich einer ethischen Analyse unterziehe. Darüber hinaus steht jedoch eine Diskussion auch zu den anderen Anwendungsfeldern aus, so etwa zur Embryonenforschung insgesamt und zur embryonalen Stammzellforschung, zum Einsatz des Gene Editing in der somatischen Gentherapie (vor allem im Bereich des Genaustauschs bei Föten und nichtzustimmungsfähigen Personen), zum Zusammenhang zwischen somatischer Gentherapie und Keimbahntherapie und, nicht zuletzt, zur Anwendung des Gene Editing für nichttherapeutische Zwecke, wie im Enhancement.

Schlagworte: Keimbahntherapie, Enhancement, Embryonenforschung, Reproduktionsmedizin, Elternschaft.

\section{Genome Editing und Embryonenforschung}

Derzeit ist das Genome Editing in vielen Bereichen noch genetische Grundlagenforschung. Sofern diese die embryonale Forschung einbezieht, treibt sie die Forschung an menschlichen Embryonen noch einmal ein Stück weiter voran und zeigt einmal mehr, dass die sogenannte verbrauchende und fremdnützige Forschung am Menschen Embryonen wenn nicht theoretisch, dann doch praktisch vom Schutz der Menschenwürde und -rechte ausschliesst. Dies sollte durch die internationalen und regionalen Rechtsinstrumente zur Bioethik und Genforschung zwar verhindert werden [1-4]; die letzten Jahrzehnte haben aber gezeigt, dass die Embryonenforschung einen grundlegenden Wertewandel in Gang gesetzt hat, der kaum rückholbar ist. Menschliche Embryonen werden heute in der Forschung weitgehend als Biomaterial angesehen, dem nur rhetorisch ein besonderer Status zuerkannt wird. Dieser Wertewandel ist vielleicht ein Grund dafür, dass die Abkehr vom Tabu der bisher sogenannten Keimbahntherapie keinen öffentlichen Aufschrei ausgelöst hat. Vielleicht haben sich aber auch nur die bioethischen Debatten im Laufe der Zeit und angesichts der Probleme in den anderen Konfliktfeldern der Gegenwart abgenutzt. Dies ist ein Fehler, der unter Umständen weitreichende Folgen haben wird.

Forschung an menschlichen Embryonen ist sowohl für die Grundlagenforschung als auch für die sogenannte Anwendungsforschung wichtig. Embryonenforschung hilft, die assistierte Reproduktionsmedizin zu verbessern; sie ist die Voraussetzung zu einem besseren Verständnis der Embryonalentwicklung im Allgemeinen und von Krankheiten im Besonderen. In den letzten Jahren ist in der Grundlagenforschung viel zur Epigenetik, das heisst zum Zusammenspiel genetischer Faktoren mit anderen Einflüssen (z.B. Infektionen, Stress, Chemikalien oder anderen toxischen Stoffen, Viren u.a.m.), gearbeitet worden [5, p. 6]. Für die klinische Medizin ist diese Forschung ausgesprochen wichtig, denn die meisten Behinderungen bzw. Krankheiten sind multifaktoriell und treten in einer grossen Bandbreite von Ausprägungen auf, und niemand kann zum Zeitpunkt des Embryonalstadiums vorhersagen, wie sich die genetischen Faktoren tatsächlich später ausprägen werden. Diejenige Grundlagenforschung, die sich auf monogenetisch bedingte Krankheiten wie Mukoviszidose, Tay Sachs oder Muskeldystrophie bezieht, setzt aber bei solchen Mutationen an, für die einzelne Gene ursächlich verantwortlich sind - dies sind die Krankheiten, die am leichtesten zu identifizieren sind.

\section{Grundlagenforschung ist eng mit der Anwendungs- forschung verzahnt}

Die Grundlagenforschung zum Genome Editing ist die Voraussetzung und zum Teil explizit ein erster Schritt auf dem Weg zur reproduktiven Anwendung, das heisst, sollten sich die Forschungsergebnisse an Tierversuchen und die ersten Embryonenversuche am Menschen betätigen, ist der Weg zum Transfer genetisch veränderter Embryonen in den Körper von Frauen gebahnt. Tatsächlich ist der Austausch mitochondrialer DNA, der seit 2015 in Grossbritannien erlaubt ist, bereits die erste Keimbahnveränderung. Die wissenschafts- und sozialethischen Diskussionen sind daher jetzt zu führen, und Regulierungen müssen möglichst bald international vereinbart werden. Weil in diesem Bereich Grundlagenforschung und klinische Anwendung so eng verzahnt sind, habe ich mich auf dem 
«Gipfeltreffen» der US-amerikanischen National Academy of Science im Dezember 2015 nicht nur für ein Verbot der klinischen Anwendung der Keimbahnveränderung, sondern darüber hinaus für ein zweijähriges Moratorium für die Grundlagenforschung mit menschlichen Embryonen ausgesprochen, sofern sie das Genome Editing am Menschen betrifft [6]. Mit diesem Vorschlag wollte ich gewährleistet sehen, dass die Staatengemeinschaft Zeit für öffentliche Diskussionen und für internationale Regulierungen bekommen. Für die anwesenden Forscher und Forscherinnen war dieser Vorschlag nicht nur undenkbar, sondern ein direkter Angriff auf ihre Forschung - als dürfe sich die ethische Reflexion nur im Rahmen der von der jeweils betroffenen Forschung gezogenen Grenzen bewegen. Das Organisationsgremium beschloss in ihrem Abschlussstatement, die öffentliche Diskussion voranzutreiben aber es stellte auch fest, dass es weiterer Grundlagenforschung bedarf und die klinische Anwendung so lange unverantwortlich sei, bis die Sicherheit und Effektivität der Methode gewährleistet sei und es einen breiten gesellschaftlichen Konsens gebe [7]. Nur kurze Zeit später wurde in Grossbritannien die erste Keimbahntherapie mit dem Austausch von mitochondrialer DNA zugelassen. Im März 2017 wurde die Erlaubnis des Einsatzes zum ersten Mal an eine Klinik in Newcastle/UK vergeben.

\section{Expertenkommissionen äussern sich positiv zur reproduktiven Keimbahntherapie}

Im Laufe der letzten anderthalb Jahre haben sich verschiedene Forschungsgesellschaften für die Genomveränderung und ihre klinische Anwendung ausgesprochen, wenngleich keine einzige Stellungnahme sich für die Anwendung der reproduktiven Keimbahntherapie zum jetzigen Zeitpunkt ausspricht. Im bisher umfassendsten Bericht der Kommission von Mitgliedern der US National Academy of Science und National Academy of Medicine vom Januar 2017 wurde das Positionspapier des Gipfeltreffens von 2015 jedoch in einem erheblichen Mass korrigiert [8]. Nach wie vor sollen zwar weitere Forschungsergebnisse abgewartet werden und «strenge Regeln» gelten, aber grundsätzlich solle, so der Bericht, das Genome Editing für einige schwerwiegende («serious») genetisch bedingte Krankheiten erlaubt werden, sofern es keine annehmbare Alternativen («reasonable alternatives») für die Paare gebe (p. 5-6). Der Bericht der NAS-Kommission schliesst nicht nur die genetische Veränderung von Embryonen, sondern auch von Spermien oder Eizellen ein, und auch die Klonierung und Generierung von Keimzellen aus Stammzellen sind vorstellbar. Interessant ist der Bericht aber vor allem wegen seiner ausführlichen ethischen Argumentation - sie wird voraussichtlich die Grundlage für die weiteren Diskussionen bilden und bedarf daher einer kritischen Prüfung.
Dass meine Position, die für die Aufrechterhaltung des Verbots der Keimbahntherapie steht, auf so erbitterten Widerstand stösst, macht stutzig. Denn meine Position spiegelt den noch bis vor kurzem geltenden internationalen Konsens und die internationalen Richtlinien, die in den vergangenen Jahrzehnten erstellt wurden. Diese hatten zur Folge, dass in vielen Ländern die Keimbahnveränderung gesetzlich verboten ist. Auch das National Institute of Health (NIH) betonte 2015, dass das Genome Editing am Menschen mit öffentlichen Mitteln nicht gefördert wird. Wie kann es zu einer solchen Erosion ethischer und rechtlicher Standards kommen? Im ethischen Diskurs, so will ich kurz zeigen, kommen zwei entscheidende Strategien zum Tragen. Zum einen werden kritische Argumente verzerrt dargestellt; und zum anderen werden die ethischen Frage so enggeführt, dass eine grundsätzliche Diskussion kaum möglich ist.

In der ethischen Diskussion scheint häufig bereits vor jeder Äusserung festzustehen, dass die Kritik an der derzeitigen Praxis der Reproduktionsmedizin ausschliesslich von konservativer, oder noch schlimmer, von religiöser Seite kommen muss. So argumentierte etwa John Harris auf dem Gipfeltreffen in Washington, dass es überhaupt nur drei Argumente gegen die Keimbahntherapie geben könne: Erstens gebe es die Position, Keimbahnzellen (!) seien «heilig und unantastbar». Aber entgegen Harris' Annahme vertritt diese Position kaum jemand; der Streit geht vielmehr darum, ab wann menschlichen Embryonen ein moralischer Schutzstatus zukommt. Zweitens bezögen sich Kritiker auf die Einschätzung, dass die Risiken für zukünftige Generationen unakzeptabel seien. Aber wie die Stellungnahmen zeigen, teilen Wissenschaftsgesellschaften diese Einschätzung, und sie ist im Übrigen medizinethisches Prinzip - Risiken müssen so gering sein, dass medizinische Eingriffe gerechtfertigt erscheinen. Harris unterstellt den Kritikern jedoch implizit, dass sie so risikoavers seien, dass ihre Abwehrhaltung nahezu jede innovative klinische Anwendung unmöglich mache. Belege für eine solche Position gibt Harris allerdings nicht. Drittens bemängelten Kritiker laut Harris, dass keine Zustimmung von zukünftigen Generationen eingeholt werden könne. Aber so, wie Harris es formuliert, ist dies ein absurdes Argument, denn kein zukünftiges Kind kann seiner Zeugung bzw. den Umständen seiner Zeugung zustimmen. Aber auch in diesem Fall verzerrt Harris das Argument und biegt es so zurecht, dass die Gegeneinwände jede Plausibilität verlieren eine beliebte, aber deshalb nicht angemessene Strategie, mit Gegenargumenten umzugehen. ${ }^{1}$ Eine solche

1 «Objections to gene editing in embryos are centred on three fallacious objections. The first is that gene editing is wrong because it affects future generations, the argument being that the human germline is sacred and inviolable. The second is that it constitutes an unacceptable risk to future generations. Third, that the inability to obtain the consent of those future generations means we must not use gene editing» [9]. 
Hermeneutik des Verdachts, die Vertreter einer konstruierten Gegenposition einmal als religiös verwurzelt und daher nur begrenzt konsensfähig, ein anderes Mal als innovationsfeindlich und, wenn dies nicht hilft, als inkonsistent hinstellt, verkennt nicht nur die Pluralität der kritischen Positionen innerhalb der Ethik, sie ignoriert auch geflissentlich alle nicht religiösen Diskussionen. So wird die Reproduktionsmedizin etwa auch innerhalb der «progressiven» feministischen Ethik, in den Disability Studies, in den Sozialwissenschaften und auch in der politischen Theorie kontrovers und kritisch begleitet.

Eine weitere Diskursstrategie betrifft die Verengung des Diskurses auf medizinethische Fragen, mit der Folge, dass der weitere Kontext der Forschung, Wissenschaft und medizinischen Praxis nahezu gänzlich ausgeblendet wird. Es bedarf nämlich einer eigenen wissenschaftsethischen, wirtschaftsethischen und politisch-ethischen Analyse, wie Wissenschaft derzeit gesteuert und gefördert wird, welche Interessen hinter den privatwirtschaftlichen Forschungsinstituten und Reproduktionskliniken stehen und welche politischethischen Hintergrundannahmen implizit vorausgesetzt werden, anstatt sie zur Diskussion zu stellen. Eine solche umfassende Analyse kann ich weder hier leisten, noch kann sie ohne die Kompetenz der Geistes-, Wirtschafts- und Sozialwissenschaften erfolgen. Sicher ist aber, dass der wissenschaftspolitische und wissenschaftsökonomische Kontext der internationalen Forschung zum Genome Editing nicht ignoriert werden darf. Derzeit liefern sich nämlich die verschiedenen Forschungsinstitute ein Wettrennen um wissenschaftliche Erfolge sowie um Lizenzen und Patente, die zuweilen über den Fortbestand der universitären sowie privaten Forschungsfinanzierung entscheiden. Und auch die privatwirtschaftlichen reproduktionsmedizinischen Angebote folgen keineswegs nur den medizinischen Erfordernissen bzw. medizinethischen Prinzipien, sondern sie folgen zumindest zu einem Teil den Prinzipien der kapitalistischen Marktwirtschaft. Dieser Kontext muss Bestandteil der Auseinandersetzung sein und darf nicht aus dem ethischen Diskurs ausgeblendet werden.

\section{Auf dem Weg zur reproduktiven Keimbahntherapie?}

Der US-amerikanische Bericht der NAS sieht es als gerechtfertigt an, Paaren, die keine «annehmbare Alternative» haben, eine Option zu geben, wie sie bestimmte vererbbare Krankheiten oder Behinderungen für immer aus dem Genom ihrer Nachfahren verbannen können. Der Bericht beginnt das Kapitel zur Keimbahntherapie mit folgenden Zahlen: Es gebe Tausende von Mutationen auf einzelnen Genen, und etwa $5 \%$ bis $7 \%$ der Bevölkerung seien davon betroffen - für einige Familien könne die Genomtherapie Entlastung von ihren «emotionalen, finanziellen und anderen Belastungen» bedeuten. Diese Beschreibung zeichnet das Bild von Paaren, die beträchtlich unter den Gendefek- ten leiden - aber es ist eine radikale Überzeichnung der medizinischen Kontexte, in denen die Keimbahntherapie angewendet werden könnte. Krankheitsbilder, die oft angeführt werden, sind die Huntington-Krankheit, das Tay-Sachs-Syndrom oder die Duchennsche Muskeldystrophie; genannt wird aber zum Beispiel auch das genetisch bedingte erhöhte Risiko, an Brustkrebs zu erkranken. Letzteres fällt nicht in die Kategorie von Krankheiten, sondern ist eine genetische Disposition für eine Krankheit. Die Liste von Genmutationen, die für jeden einzelnen Entwicklungsschritt der Reproduktionsmedizin angeführt wird, ist immer gleich - nicht, weil die genannten Krankheiten lebensweltlich so plausibel sind, sondern weil sie auf Einzelgenmutationen beruhen. Für die Zystische Fibrose etwa werden derzeit mit der gleichen Technik somatische Gentherapien entwickelt; diese stehen aber vor besonderen Herausforderungen, so die Kommission, weil verschiedene Zelltypen von der Krankheit (u.a. Lungen- oder Leberzellen) betroffen sind.

\section{Die ethische Diskussion der reproduktiven Keimbahntherapie}

\section{Der «Fall» der Zystischen Fibrose - eine ethische Analyse}

Für die Notwendigkeit der embryonalen Genveränderung wird oft die Krankheit Mukoviszidose bzw. Zystische Fibrose (CF) angeführt, die ich hier als exemplarisches Beispiel der Keimbahntherapie näher betrachten will. Zystische Fibrose kommt in Europa und den USA vor allem in der «kaukasisch» bezeichneten Bevölkerungsgruppe erhöht vor. Die US-amerikanische Cystic Fibrosis Foundation gibt das Risiko für die ethnischen Gruppen mit diesen Zahlen an: Das Risiko, sogenannter Träger des Gens (ohne Krankheitsauswirkungen) zu sein, ist

«-1 in 29 Caucasian-Americans

-1 in 46 Hispanic-Americans

-1 in 65 African-Americans

-1 in 90 Asian-Americans».

Das Risiko, an CF zu erkranken, wird so geschätzt: «-1 in 2500-3500 Caucasian-Americans

-1 in 4000-10000 Hispanic-Americans

-1 in 15000-20000 African-Americans

-1 in 100000 Asian-Americans». ${ }^{2}$

Weil die Krankheit autosomal rezessiv ist, setzt dieser «Fall» zwei Personen voraus, die beide an Mukoviszidose erkrankt sind und die unbedingt ein genetisch

2 Die Stiftung bezieht sich auf Zahlen von 2004. Abrufbar unter: www.cff.org/What-is-CF/Testing/Carrier-Testing-for-CF/ (Zugriff: 2.5.2017). In Grossbritannien sind ca. 9000 Menschen betroffen, in den USA 30000 , und weltweit 90000 [10]. Das Interesse an CF mag daher auch daran liegen, dass es die am weitesten verbreitete monogenetische Krankheit in den Ländern des «Nordens» ist. 
verwandtes Kind bekommen wollen. Ihnen, so sagen die Wissenschaftler, könnte nicht die Präimplantationsdiagnostik, wohl aber die Keimbahntherapie helfen. Das Szenario verliert jedoch seine Plausibilität, sobald es aus der isolierten Perspektive der Genetik, dem «Laborblick», in seinen «normalen» gesellschaftlichen «Sitz im Leben» zurückverlegt wird: Die durchschnittliche Lebenserwartung für Menschen mit CF beträgt derzeit ca. 37 Jahre. Der NAS-Bericht sagt, dass es aufgrund der steigenden Lebenserwartung immer mehr Personen geben wird, die sich zum Beispiel in Selbsthilfegruppen kennenlernen würden - dass die genannte Konstellation eintreten kann, wird daher niemand bestreiten. Viele betroffene Männer sind aber zeugungsunfähig oder nur eingeschränkt zeugungsfähig, was die Erfolgsquote der In-vitro-Fertilisation aller Voraussicht nach negativ beeinflusst. Frauen haben eine etwas geringere Lebenserwartung als Männer, für beide liegt diese jedoch in der Phase der Familiengründung. ${ }^{3}$ Das angeführte Paar würde, wenn es nicht bereits vor dem Erreichen des 20. Lebensjahres eine Familie gründete, ihr Kind voraussichtlich vor dessen Volljährigkeit als Vollwaise hinterlassen. Wenn wir das Beispiel von diesem Blickwinkel aus ansehen, sind familiäre Tragödien geradezu vorprogrammiert.

\section{Das Argument der reproduktiven Autonomie}

Was im Bericht als unhintergehbar hingenommen wird, sind die unmittelbaren Umstände der reproduktiven Entscheidungen, während alle mittelbaren biographischen Umstände ausgeblendet werden. Was ethisch zählt, ist einzig der Wunsch nach einem genetisch verwandten Kind. Nur wenn der Wunsch beider an Mukoviszidose erkrankten Personen nach der gemeinsamen, mütterlichen und väterlichen genetischen Verwandtschaft absolut gesetzt wird, erscheint nämlich die Keimbahntherapie als «letzte Option» (im Bericht werden die Begriffe «last option» und «last resort» verwendet). Diese Argumentation der Kommission ist aber eine Irreführung, weil die Reduktion auf die «letzte Option» eine Binsenweisheit enthält: Wenn alle anderen Alternativen ausgeschlossen werden, bleibt am Ende in der Tat nur eine einzige Option übrig. Sie als faktisch «letztes Mittel» zu bezeichnen, ist ethisch falsch - das Urteil basiert nämlich auf der Wertannahme, dass die genetische Herkunft ein so hohes Gut ist, dass es alle anderen ethischen Gesichtspunkte überbietet. Was von Wissenschaftlern als letzte Option für die «emotionale, finanzielle und anderweitige Belastung» der Paare dargestellt wird, erweist sich als medizinisch, biographisch und ethisch sehr viel komplexer und angesichts alternativer Optionen keineswegs naheliegend. Die NAS-Kommission führt folgende Gründe an, warum sie die Wünsche der in Frage kom-

3 Laut einer Studie mit über 30000 Betroffenen liegt die durchschnittliche Lebenserwartung von Frauen bei 36 Jahren, von Männern bei 38,7 Jahren [11] menden Gruppe zukünftiger Eltern für unhintergehbar hält: Viele Paare wollen unbedingt ein genetisch verwandtes Kind; einige Paare haben ethische Probleme mit der Präimplantationsdiagnostik, weil diese die Selektion und Verwerfung von Embryonen impliziert. Und einige Paare lehnen den Schwangerschaftsabbruch als grundsätzliche Alternative zur Keimbahntherapie ab. Für all diese Paare, so sagt die Kommission, wäre die Keimbahntherapie eine Alternative. Allerdings darf mit einigem Recht gefragt werden, warum die beiden letztgenannten Gruppen ausgerechnet einer Genomveränderung ihres Kindes zustimmen sollten, die doch voraussichtlich ebenfalls den «Verbrauch» von menschlichen Embryonen erforderlich macht. Es kann daher nicht ausgeschlossen werden, dass hier Fälle und Fallkonstellationen konstruiert werden, deren lebensweltliche Relevanz durchaus bezweifelt werden kann.

Manche Befürworter in Medizin, Recht und Ethik vertreten eine inzwischen allzu bekannte Argumentation, die aber durch Wiederholung nicht plausibler wird. Diese besagt, dass Paaren nicht vorgeschrieben werden kann, welche der innerhalb der Reproduktionsmedizin angebotenen Methoden sie wählen «dürfen», um ein Kind zu bekommen - im Fall des erwähnten Paares wäre zum Beispiel die Spermienspende eine empirische und ethisch mögliche Alternative. Wir führen hier aber eine antizipatorische Diskussion, denn es geht ja darum, ob die Forschung der reproduktiven Keimbahntherapie erlaubt werden soll oder nicht. Damit wird die Abwägung nicht als eine zwischen verschiedenen Forschungsinteressen dargestellt, sondern als Abwägung zwischen den reproduktiven Rechten der prospektiven Eltern einerseits und dem Recht des Staates, diese Rechte einzuschränken, andererseits. Dies ist eine klassische liberale Position, die auf die Debatte um Frauenrechte und ihre Reproduktionsrechte zurückgeht, die ich in anderem Kontext durchaus teile. ${ }^{4}$ Sie wird hier angeführt (oder benutzt), um Forschungsinteressen zu rechtfertigen, ohne dass andere Interessen wie die wissenschaftliche Reputation oder wirtschaftliche Interessen benannt werden.

Sobald der Blick von der präferenzutilitaristischen Voraussetzung abweicht, die Reproduktionsmedizin habe den Präferenzen der zukünftigen Eltern prima facie zu folgen, verändert sich die ethische Fragestellung grundlegend. Dies sieht auch die NAS-Kommission so. Im Mittelpunkt der ethischen Reflexion muss die Diskussion stehen, welche Optionen des Kinderwunsches «annehmbar», «vernünftig» oder «rechtfertigbar» sind - der Begriff «reasonable», den die NASKommission wählt (p. 6), lässt offen, welcher Lesart wir hier folgen sollen. In der liberalen ethischen Argumentation, die ähnlich auch vom Nuffield Council und anderen Stellungnahmen vertreten wird, ist nun nicht

4 Die Position zu teilen, heisst nicht, einer liberalen Ethik unkritisch $\mathrm{zu}$ folgen: Vgl. [12]. 
die Forschung, sondern der Staat grundsätzlich in der Begründungspflicht, wenn er Freiheiten einschränkt: Der einzige Grund, der als Rechtfertigung für die Einschränkung der reproduktiven Autonomie anerkannt wird, ist der ethische Konflikt zwischen elterlichen Rechten einerseits und Rechten der Kinder andererseits. Aber längst nicht alle Staaten teilen diese libertäre, politisch-ethische Argumentation. Es ist Aufgabe der Ethik, diese Voraussetzung deutlich zu machen und sie mit alternativen Deutungsrahmen der Forschungsethik und -politik zu konfrontieren. Weder die USA noch die EU vertreten eine solche libertäre politische Ethik.

\section{Der Wunsch nach einem genetisch verwandten Kind ist ein verständliches Motiv, nicht aber ein rechtferti- gender Grund für die reproduktive Keimbahntherapie}

Assistierte Reproduktionsmedizin setzt das Handeln gesellschaftlicher Institutionen, d.h. der Forschung und Medizin, voraus. Daher unterliegen die Interessen und Rechte der Klienten und Klientinnen der Reproduktionsmedizin in besonderer Weise der Abwägung mit anderen Gütern sowie den Rechten anderer. Nun ist ein verständliches und von vielen geteiltes Motiv, nämlich der elterliche Wunsch, ein genetisch verwandtes Kind zu bekommen, noch kein rechtfertigender Grund für die Erforschung der reproduktiven Keimbahntherapie, wenn die Rechte anderer dabei zur Disposition stehen. Es geht hier um zwei Rechte zukünftiger Kinder, um ihr Gesundheitsrecht und um ihr Freiheitsrecht. Letzteres meint das Recht der zukünftigen Kinder auf ihren Spielraum der Freiheit und, unter anderem, um ihre reproduktive Freiheit. Es geht also nicht, wie Harris meint, um die Zustimmung zu ihrer Zeugung. Im oben geschilderten Fall der Zystischen Fibrose, der sich auf andere Konstellationen übertragen lässt, kann man zudem fragen, ob das Recht auf Wohlergehen und Fürsorge eines Kindes missachtet wird, wenn die Eltern wissen können, dass ihre Kinder voraussichtlich noch in der Phase der Kindheit oder Jugend Vollwaisen werden

Die NAS-Kommission folgt im Grundsatz einer solchen Interpretation der Abwägung von Rechten. Erstens empfiehlt sie eine Eingrenzung der Keimbahntherapie auf «schwerwiegende genetisch bedingte Krankheiten». Und zweitens anerkennt sie die Gesundheitsrisiken für die zukünftigen Kinder und sieht es als gerechtfertigt an, die elterliche Freiheit einzuschränken, solange die Risiken für die Kinder nicht auf ein Mindestmass reduziert sind. Die Sicherheit der Keimbahntherapie ist entsprechend auch für eine liberale Position die Conditio sine qua non für die Begründung (und Rechtfertigung) der klinischen Anwendung. Aber die angeführten Kriterien sind bei näherem Hinsehen nicht plausibel: Erstens gibt es, wie gesagt, für alle Fallkonstellationen Alternativen, sofern nicht die genetische Verwandtschaft absolut gesetzt wird, und zwar Alternativen innerhalb der assistierten Reproduktionsmedizin. Zweitens werden die Kriterien für die Einschränkung kaum lange währen, wenn bereits der Wunsch nach einem genetisch verwandten Kind als Recht betrachtet wird; eine Ausweitung ist geradezu vorprogrammiert, weil nicht zwischen Wünschen und Rechten unterschieden wird [13]. Und dies klingt allzu bekannt für diejenigen, die die letzten Jahrzehnte der bioethischen Diskussion verfolgt haben. Es geht dabei nicht um das Argument der «schiefen Bahn», sondern vielmehr darum, dass Kriterien so vage formuliert sind, dass sie sowohl eine enge als auch eine weite Interpretation erlauben. Der NAS-Bericht ist ein Beispiel dafür, dass eine enge Interpretation suggeriert wird, die später jedoch zurückgenommen und unter Rückgriff auf die reproduktive Freiheit korrigiert werden kann.

\section{Freiheitsrechte betreffen nicht nur Eltern, sondern auch Kinder}

In der bisherigen Diskussion werden Elternrechte vor allem als reproduktive Freiheitsrechte diskutiert, die als Recht auf ein genetisch verwandtes Kind begriffen werden. Kinderrechte werden demgegenüber fast ausschliesslich als Gesundheitsrechte betrachtet. Wie das Beispiel der genetischen Disposition zur Brustkrebserkrankung zeigt, die in Zukunft für eine Keimbahntherapie qualifizieren könnte, umfasst das kindliche Recht auf Gesundheit explizit Risikofaktoren und spät ausbrechende Krankheiten wie Chorea Huntington. Gegenüber diesen Gesundheitsrechten - die in der ethischen Diskussion durchaus kontrovers diskutiert werden - wird die Einschränkung der Freiheitsrechte der Kinder gar nicht oder nicht mit der gleichen Gewichtung thematisiert. Dies ist ein folgenreicher Fehler. Denn es ist nicht nur das allgemeine Freiheitsrecht zukünftiger Generationen, sondern - und das ist neu im Fall der Keimbahntherapie - das reproduktive Freiheitsrecht der Kinder, das die Genomveränderung auch für eine liberale Argumentation problematisch machen muss. Den Kindern wird nämlich eine schwerwiegende Belastung auferlegt, wenn ihre Handlungsfreiheit, u.a. auch ihre reproduktive Freiheit, eingeschränkt wird. Weil sie Kinderrechte nicht als Freiheitsrechte sieht, kommt die NAS-Kommission zum Schluss, dass der elterliche Wunsch nach genetischer Verwandtschaft schwerer wiegt als die Einschränkung der Freiheit des zukünftigen Kindes. Sie gibt damit einerseits der elterlichen Freiheit Vorrang vor der kindlichen Freiheit, und sie gibt der Gesundheit eines Kindes Vorrang vor dessen Handlungsfreiheit. Beide Urteile sind aber eigens $\mathrm{zu}$ rechtfertigen, was nicht geschieht. Warum aber müssen die kindlichen Freiheitsrechte in den Blick genommen werden?

Die NAS-Kommission fordert ausdrücklich eine Langzeitüberwachung der Kinder, und zwar unabhängig vom Recht der Kinder, die Teilnahme an lebenslangen wissenschaftlichen Studien ablehnen zu können. Sie rät dazu, Eltern und Kinder die Teilnahme zu empfehlen, und setzt auf den sanften Druck solcher Empfehlungen. Die NAS-Kommission fordert, dass vor der kli- 
nischen Einführung ein Plan erstellt wird, wie mit den Langzeitstudien umgegangen werden soll. Aber sie gibt keinerlei Kriterien an die Hand: Sollte die Zustimmung zu einer Langzeitstudie zur Bedingung der Keimbahntherapie gemacht werden oder nur, wie die Kommission meint, dringend empfohlen werden? Oder sollte den Eltern nur gesagt werden, dass sie im Falle eines «opt out» das Risiko für etwaige Gesundheitsfolgen ihrer Kinder alleine tragen werden? Was heisst dies dann für etwaige Versicherungen? Die Teilnahme an Entwicklungsstudien nach Genomveränderung unterscheidet sich von anderen Langzeitstudien. Es geht ja, wie gesagt, nicht nur um die gesundheitliche Entwicklung der Kinder, wie dies auch in der herkömmlichen Reproduktionsmedizin der Fall ist, sondern die Studien betreffen darüber hinaus auch die reproduktiven Entscheidungen der Kinder und die Gesundheit der zweiten Generation. Wir greifen nicht nur massiv in das Genom oder die Keimbahn ein, sondern ebenso greifen wir in die Lebensentscheidungen von zukünftigen Generationen ein. Aus Sicherheitsgründen wird den Kindern zum Beispiel empfohlen werden müssen, auch selbst Kinder über die assistierte Reproduktion zu bekommen. Selbst wenn Betroffene das Recht haben, die Teilnahme an den Studien abzulehnen, ist das Wissen über ihre genetische Modifikation genauso wenig rückholbar wie die Modifikation selbst, und es wird ihre reproduktive Freiheit wegen der generationsübergreifenden genetischen Effekte beeinflussen.

Welche Freiheitsrechte haben Priorität - die der Eltern oder die der zukünftigen Kinder? Es ist die Aufgabe der öffentlichen ethischen Diskussion und letztlich Aufgabe des Staates (oder der Staatengemeinschaft), den Schutz und die Rechte aller Betroffenen zu gewährleisten. Es kann nicht sein, dass die Rechte zukünftiger Kinder nur eine Nebenrolle in dieser ethischen Diskussion spielen - denn Eltern, deren Freiheit in der Tat respektiert werden muss, sind zugleich mit ihrer Freiheit auch verantwortlich für ihr Handeln. Sie müssen ihre Entscheidungen im Hinblick auf das Wohlergehen und die Freiheit ihrer Kinder treffen, und es ist Sache des Staates, die Rahmenbedingungen zu schaffen, innerhalb deren dies möglich ist [13]. Und wenn die NASKommission grundsätzlich der Meinung ist, die elterliche Freiheit könne aus ethischen Gründen eingeschränkt werden, dann stellt sich die Frage, warum dies nur insoweit geschieht, als die jeweiligen Empfehlungen die Zulassung der Keimbahntherapie nicht gefährden. Rechtfertigen die - wie oben gezeigt - lebensweltlich geradezu bizarren und konstruierten Fälle wirklich die Abkehr vom Verbot eines irreversiblen Eingriffs in das menschliche Genom? Oder ist es nicht vielmehr irreführend, die Optionen ausschliesslich innerhalb der Grenzen der genetischen Verwandtschaft zwischen beiden Eltern und dem zukünftigen Kind zu diskutieren? Stellen wir diese Vorentscheidung für einen Moment in Frage, erweist sich die ethische Begründung für die Keimbahntherapie, sie sei das «letzte
Mittel» in ansonsten tragischen Fällen, selbst innerhalb der assistierten Reproduktionsmedizin als falsch. Im Gegenteil - dieses Urteil erweist sich als eine nicht weiter begründete Absolutsetzung der genetischen Verwandtschaft, oder aber, und das wäre schlimmer, als eine rhetorische Rechtfertigung einer Forschungsrichtung, indem mit dem Leid von Paaren argumentiert wird, die von einem unerfüllten Kinderwunsch betroffen sind. Ihnen und der Gesellschaft wird suggeriert, die Genetik habe nun endlich das Zaubermittel gefunden, um genetisch bedingte Krankheiten im «Keim» auszurotten. Damit aber wird der Mythos einer modernen, liberalen Eugenik beschworen, der mit der Realität der Forschung und klinischen Anwendung wenig zu tun hat.

\section{Folgen der Keimbahnveränderung}

Ethisches Urteilen gibt es nicht ohne die Frage nach den Folgen einer Handlung oder Praxis. Gehen wir also einmal davon aus, wir würden die embryonalen Genomtherapie zulassen. Die Befürworter sagen, dass die Genomtherapie die Präimplantationsdiagnostik und das Verwerfen von Embryonen, womöglich sogar spätere Schwangerschaftsabbrüche reduziert. Gerade diejenigen, die Probleme mit der Präimplantationsdiagnostik und dem Schwangerschaftsabbruch haben, müssten daher die Erforschung der Keimbahntherapie befürworten. Dieses Argument ist auf die «konservativen» Gruppierungen gemünzt. Diese Prognose ist aber reine Spekulation. Wer Genomtherapie in der Reproduktionsmedizin will, will auch die Präimplantationsdiagnostik, und eine liberale Position wird davon ausgehen, dass die Entscheidung über einen Schwangerschaftsabbruch in jedem Fall bei der Frau liegt und daher nicht präjudiziert werden darf. Ob die absolute Zahl der verworfenen Embryonen aber tatsächlich zurückgehen würde, kann heute niemand sagen. Weder wissen wir, wie gross der Bedarf ist, noch wissen wir, wie viele der betroffenen Paare sich für die Präimplantationsdiagnostik entscheiden würden. In der klinischen Anwendung der Keimbahntherapie wäre es unverantwortlich, die genetisch veränderten Embryonen nicht vor der Implantierung auf nichtintendierte genetische oder chromosomale Veränderungen, also auf sogenannte «off-target»-Effekte, zu untersuchen. Wer der Genomveränderung zustimmt, wird auch der medizinisch indizierten Diagnostik der Embryonen zustimmen. Nehmen wir nun weiter an, die Embryonen würden tatsächlich implantiert, so werden die meisten Frauen unter diesen hochexperimentellen Bedingungen sicher auch einer Pränataldiagnostik zustimmen auch diese müsste aus Verantwortungsgründen empfohlen werden. Zukünftige Eltern müssen dies zumindest wissen, bevor sie der Keimbahntherapie zustimmen. Darüber hinaus müssen Eltern bereits zu Beginn der Behandlung zustimmen, dass ihre Kinder an einer potentiell lebenslangen Langzeitstudie teilnehmen. Die Forschung braucht diese Informationen, 
um die Daten zu gewinnen, die für die Sicherheitsprognosen unabkömmlich sind. Ob die Genomveränderung wirklich sicher ist, wird sich erst in der zweiten oder dritten Generation zeigen, und das heisst, dass wir erst in der zweiten Hälfte dieses Jahrhunderts wissen werden, ob sie als Therapie genetisch bedingter Krankheiten erfolgreich ist oder nicht zu viele andere genetische Mutationen auslöst. Um dies aber kontrolliert prognostizieren zu können, werden genügend Teilnehmer an den Langzeitstudien gebraucht. Was sich im Labor vielleicht kohärent anhört, weil keine Freiheitsrechte von Zellkulturen oder Versuchstieren betroffen sind, stellt sich im wirklichen Leben von Menschen vollkommen anders dar. Eine Sicherheitsprognose ist ohne die freiwillige und informierte Zustimmung der Eltern und dann - in Zukunft - ohne die Zustimmung der Kinder selbst nicht möglich. Die Forschung ist hier in einer Zwickmühle, aus wissenschaftlichen und ethischen Gründen braucht sie Studien, um ihren eigenen Standards genügen zu können, aber aus ethischen Gründen muss sie auch die Freiwilligkeit der Betroffenen respektieren. Da die Freiheitsrechte der Kinder in jedem Fall Vorrang vor den Forschungsinteressen haben, wetten wir darauf, dass wir die Daten auf freiwilliger Basis erhalten, ohne dies garantieren $\mathrm{zu}$ können - aus Perspektive der Ethik, die an einer verantwortlichen Folgenbeurteilung nicht nur interessiert ist, sondern deren Urteil von diesem Wissen teilweise abhängt, ist dies aber nicht genug. Nun zeigen Erfahrungen aus anderen Bereichen, dass unter diesen Bedingungen finanzielle Anreize geschaffen werden, um die Betroffenen in eine bestimmte Richtung $\mathrm{zu}$ lenken - aber auch dies ist aus ethischer Sicht problematisch, weil es leicht zu Abwägungskonflikten der Betroffenen führt, die aus liberaler Sicht gerade vermieden werden müssen.

Wir respektieren also die reproduktive Freiheit der Eltern um den Preis der Einschränkung der Freiheitsrechte ihrer Kinder, und wir verwickeln uns hierbei in ethische Widersprüche, die wir nicht mehr auflösen können. Und all dies tun wir, weil wir der Begründung folgen, es gebe keine «annehmbaren Alternativen» zur genetischen Verwandtschaft, wenn es um den Kinderwunsch geht. Dies ist eine abenteuerliche und letztlich absurde Vorstellung, die allen Eltern Hohn spricht, die Kinder lieben und für sie sorgen, weil sie deren (soziale) Eltern sind - weil sie Elternschaft als Aufgabe und Geschenk, und nicht als biologisches Schicksal erfahren. Es war einmal niemand anders als die Reproduktionsmedizin, die uns unsere relative Unabhängigkeit von der Natur vor Augen führte - wir sollten uns nun darauf besinnen, dass die menschliche Elternschaft auf einer solchen relativen Unabhängigkeit nicht nur wegen der Technik, sondern auch wegen unserer Fähigkeit basiert, Verantwortung zu gestalten. Elternschaft ist nämlich weder nur biologisches Schicksal noch nur technische Herstellung, sondern sie ist vor allem eine soziale Praxis, die ethisch zu reflektieren ist.

\section{Ist die Einschränkung der reproduktiven Freiheits- rechte ethisch zu rechtfertigen?}

Darf die Gesellschaft Paaren also zumuten, den sichersten und schonendsten Weg zu einem Kind zu wählen, dessen Eltern sie sein werden, wenn nur so die Rechte ebendieses Kindes gewährleistet werden können? Ich meine Ja. Die NAS spricht von Tausenden von Mutationen und suggeriert damit, dass die Genomtherapie ein Weg für viele Paare sein könnte. In den allermeisten Fällen der $5 \%$ bis $7 \%$ von genetischen Mutationen betroffenen Menschen, von denen die NAS-Kommission spricht, wird sich die Mutation aber gar nicht auswirken. In anderen Konstellationen wird die Präimplantationsdiagnostik ein Weg sein, der Paaren empfohlen werden kann - auch wenn deren Probleme nicht kleingeredet werden sollen. Eine durchaus «annehmbare» Option für den Fall, dass beide (zukünftigen) Eltern an Mukoviszidose leiden, ist die Spermienspende. Diese Option erscheint mir für diese Konstellation nicht nur «annehmbar», sondern weitaus besser begründet zu sein als die Keimbahntherapie. Für die (in Deutschland verbotene) Eizellspende muss die ethische Diskussion eigens weitergeführt werden, ebenso wie für die Embryonenspende und letztlich auch die Adoptionspraxis. All diese Optionen sind Bestandteil dessen, was wir unter Elternschaft verstehen - und zwar unabhängig davon, ob wir genetisch verwandte Eltern sind oder nicht. Alle Beteiligten der assistierten Reproduktionsmedizin sind auf Rahmenbedingungen und Regulierungen angewiesen - deshalb ist es beklagenswert, wenn die Diskussion von einer Berufsgruppe dominiert wird. Wir brauchen endlich eine breite öffentliche Diskussion darüber, welche Wünsche in der Reproduktionsmedizin erfüllt werden sollen, welche (und wessen) Rechte gelten, welche (und wessen) Pflichten wie gewichtet werden müssen - und was Elternschaft unter den Bedingungen der assistierten Reproduktionsmedizin bedeutet.

\section{Forschungsinteressen und Freiheit der Forschung}

Für manche Forscher ist die klinische Anwendung ethisch zweifelhaft, oder aber Zukunftsmusik. Für sie tritt in der Diskussion das Forschungsinteresse und das Recht auf Forschungsfreiheit in den Vordergrund. In der Tat erscheint es zunächst einmal einleuchtend zu sein, herausfinden zu wollen, was Genome Editing zum Beispiel für die embryonale Stammzellforschung bedeutet, wie die assistierte Reproduktion verbessert werden kann, ob Keimzellen generiert werden können usw. Dies sind meines Erachtens Fragen, die diskutiert werden können und jeweils eigens erörtert werden. Aus diesem Grund habe ich mich auch nur für ein Verbot der klinischen Anwendung der Keimbahntherapie innerhalb der Reproduktionsmedizin ausgesprochen. Ein kategorisches Verbot der reproduktiven Keimbahntherapie ist die einzige ethisch zu rechtfertigende Position - alle anderen Positionen können, wie oben gezeigt, nicht garantieren, wie sie zum einen die Frei- 
heitsrechte der Kinder schützen und wie sie zum anderen die Ausweitung auf eine nichttherapeutische Anwendung verhindern können. Meine Position ist jedoch nicht kategorisch im Hinblick auf die allgemeine Embryonenforschung. Dies setzt aber voraus, dass die Keimbahntherapie bereits in der anwendungsorientierten Grundlagenforschung ausgeschlossen wird. $\mathrm{Ob}$ das Genome Editing in der Embryonenforschung notwendig ist, ist dann eine andere Debatte - diese Diskussion muss aber unabhängig von der Keimbahntherapie geführt werden.

\section{Sozialethische Effekte - die Veränderung von Elternschaft}

Die Genomveränderung verändert nicht nur die Gene, sondern auch das soziale Verständnis von Elternschaft. Setzen wir einmal voraus, dass die Genomtherapie sicher wäre und in Zukunft als eine Option unter anderen in der Reproduktionsmedizin angeboten würde ein Szenario, das durchaus realistisch erscheint. Die NAS-Kommission räumt ein, dass ihre Kriterien vage sind. Was eine «schwerwiegende» Krankheit ist, sei im klinischen Kontext zu klären; wo die Grenze zwischen Therapie und Enhancement verläuft, mag die Kommission nicht entscheiden. Aber wenn niemand entscheidet, dann gilt die «normative Kraft des Faktischen», nämlich die Machbarkeit nach Massgabe der modernen Forschung und Reproduktionsmedizin. Nun hat in den letzten Jahrzehnten bereits die assistierte Reproduktionsmedizin, die zeitgleich mit der Gendiagnostik eingeführt wurde, das Verständnis von Elternschaft grundlegend verändert. Elternschaft schlägt immer mehr von der Annahme und Anerkennung eines Kindes als dem nicht planbaren «Anderen» oder «Neuen» in einen reflexiven Wunsch um, dessen Erfüllung geplant und gegebenenfalls mit Hilfe der Medizin umgesetzt werden kann. Häufig prallen die nachvollziehbaren Wünsche nach einem gesunden Kind allerdings mit aller Wucht auf die Tatsachen der autobiographischen wie auch der medizinischen Grenzen - im Scheitern von Schwangerschaften oder aber in einer pränatal diagnostizierten Behinderung werden diese Grenzen erfahren. Spätestens bei der Frage nach der Gesundheit ihres Kindes treffen die individuellen Wünsche von Eltern, ihre biographischen Bedingungen und die gesellschaftlichen Realitäten aufeinander [14]. Zukünftige Eltern können sich häufig ein Leben mit einem behinderten Kind nicht vorstellen, und alle Bemühungen der Behindertenverbände und die Inklusionsbemühungen im Gefolge der Konvention zu den Rechen von Menschen mit Behinderung haben dies nicht geändert. Insbesondere Frauen wissen, dass sie es sind bzw. sein werden, die für Kinder mit besonderen Bedürfnissen ihre eigenen Wünsche, Bedürfnisse und Rechte hintenanstellen müssen. Denn unsere Gesellschaften und die inzwischen weitgehend kommerzialisierte Reproduk- tionsmedizin sind zwar in der Lage, Milliardenbeträge für die Verhinderung behinderter Kinder aufzubringen, aber offensichtlich sind wir nicht willens oder in der Lage, die Rechte insbesondere von Frauen und die Rechte von Kindern mit Behinderungen so ernst zu nehmen, dass diese ein ihren Bedürfnissen und Fähigkeiten entsprechendes Leben haben können.

\section{Mehr Gerechtigkeit durch assistierte Reproduktionsmedizin?}

Setzen wir bei der gesellschaftlichen Realität ein, in welcher Menschen mit Behinderung tatsächlich vielfältig benachteiligt werden, dann verspricht die Genomtherapie von Embryonen, so einzelfallbezogen sie auch erscheinen mag, in der Tat eine Lösung für beide Seiten, Eltern und Gesellschaft gleichermassen. Aber sie transportiert damit zugleich das Bild einer Gesellschaft, die Gesundheit zur Voraussetzung eines guten und gelingenden Lebens macht. Wie schon zuvor in der impliziten Wertung der Pränataldiagnostik und Präimplantationsdiagnostik wird die Verhinderung von genetisch bedingten Behinderungen, Krankheiten und Krankheitsdispositionen zum Bestandteil dessen, was heute «verantwortliche Elternschaft» genannt wird.

Die Diskurse um die Rechte von Menschen mit Behinderung - und die Rechte ihrer Familien - sind hinlänglich bekannt; die NAS-Kommission diskutiert sie $\mathrm{zu}$ Recht unter anderem als Gerechtigkeitsfrage. Aber es überrascht, wie sie argumentiert. Die genetische Intervention in die menschliche Konstitution sei ein Weg zu einer gerechteren Gesellschaft, in der alle Mitglieder möglichst gleiche Chancen haben. Die Keimbahntherapie könne, so die Kommission, gerade einen Beitrag zur Verringerung der Gerechtigkeitslücke leisten. Eltern, so muss man die implizite (und zum Teil explizite) Botschaft der Reproduktionsmedizin interpretieren, können nicht wollen, dass ihre Kinder einen «schlechteren» Start ins Leben haben als gesunde Kinder. Weil Gerechtigkeit vor allem als Chancengerechtigkeit definiert wird, kann sie auf diese Weise individualisiert und als Frage der Technik ausbuchstabiert werden. Mit sehr viel mehr Recht könnte sie aber als Frage einer gerechteren Verteilung der Ressourcen und der sozialen Kompensation für bestehende Ungleichheiten diskutiert werden - auch im Hinblick auf die zugrundeliegende Gerechtigkeitstheorie gibt es also durchaus Diskussionsbedarf.

In der sozialethischen Betrachtung, die Rechte und Pflichten zum Ausgangspunkt nimmt, verlagern sich die Prioritäten. Zuerst müssen den Eltern ihre Ängste genommen werden, für ihre Kinder sei kein Platz in unserer Gesellschaft, und dies ist eine Frage an die Solidargemeinschaft. Den Frauen, deren reproduktive Freiheit so sehr betont wird, wenn es um die Reproduktionsmedizin geht, müssen ihre berechtigten Ängste genommen werden, dass ihre Rechte genau dann nicht mehr zählen, wenn sie ein Kind mit einer Behinderung oder Krankheit haben. Kinder mit bestimmten Gesund- 
heitsrisiken müssen in unseren Gesellschaften willkommen sein und ihre Gesundheitskosten solidarisch getragen werden - nicht deshalb, weil sie etwas «beizutragen» haben, sondern weil eine responsorische Verantwortung Kern unserer Moralfähigkeit ist. Wir können weder anderen Menschen noch uns selbst zumuten wollen, darüber zu entscheiden, welches Leben lebenswert ist und welches nicht [13]. Hinter diesem Argument steht keine verkappte theologische Mahnung. Es reicht, wenn wir uns eingestehen, dass es keinen «objektiven» oder gar empirisch nachweisbaren Wert gibt, den wir für ein Menschenleben angeben können, selbst dann, wenn wir selbstverständlich zwischen Gesundheit und Krankheit unterscheiden. Es ist verständlich, wenn Eltern, die etwa an Mukoviszidose erkrankt sind, ihren Kindern ihr eigenes Schicksal ersparen wollen und trotz ihrer genetischen Konstellation nicht auf ein Kind verzichten wollen. Die assistierte Reproduktionsmedizin antwortet auf diesen Wunsch, aber sie ist genauso dem Wohl und den Rechten der Kinder verpflichtet. Aber es bleibt dennoch letztlich eine gesellschaftliche Frage, wie wir mit denjenigen Menschen umgehen, die eine Behinderung haben - und dies haben wir normativ bereits getan, indem wir die Konvention zu den Rechten von Menschen mit Behinderung ratifiziert haben. Diese gilt es umzusetzen, nicht auszuhöhlen.

\section{Reproduktive Autonomie und Enhancement}

Könnte es sein, dass nicht die «reproduktive Autonomie» von einigen Gruppen von Eltern, sondern das Ziel, Kinder mit genetisch bedingten Behinderungen oder Krankheiten zu verhindern, das nicht ausgesprochene Motiv für die Forschung der Keimbahntherapie ist? Wäre dies so, so müsste dies auch öffentlich diskutiert werden. Teile der Wissenschaft und Ethik läuten bereits eine neue Ära der genetischen Modifikation von Menschen ein, auch wenn sie keine Krankheitsdispositionen haben. Diese Anwendung wird heute «Enhancement» genannt, aber dahinter stehen die gleichen eugenischen Visionen wie vor einem Jahrhundert und wie in der Vergangenheit, wird es auch heute Paare geben, die ihre Kinder für solche genetischen Veränderungen zur Verfügung stellen werden. Wie schnell die scientific community bereit ist, jahrzehntelang geltende ethische Standards aufzugeben, zeigt sich wiederum am Bericht der NAS-Kommission. Weil die Grenze zwischen Therapie und Enhancement fliessend sei, schliesst sie das Enhancement bereits schon jetzt nicht mehr grundsätzlich aus, sondern nur noch «zu diesem Zeitpunkt».

Wir sollten uns hüten, die Diskussion um das Genome Editing auf die Therapie oder das Enhancement zu beschränken - aber wir sollten auch nicht so naiv sein zu glauben, dass die Grundlagenforschung nicht zur Anwendung in der assistierten Reproduktionsmedizin führen wird. Früher oder später wird der schleichende Übergang von Therapie und Enhancement als Argu- ment für das Enhancement vorgebracht werden, zu verlockend sind die Früchte, die an diesem Baum der Erkenntnis hängen. Sie locken als monetärer Gewinn in Form von Patenten und Lizenzen genauso wie als wissenschaftliche Reputation. Ethische Bedenkenträgerinnen stören dieses Getriebe genauso wie eine kritische öffentliche Diskussion. Im NAS-Bericht wird für strenge Zulassungskriterien plädiert - aber eine offene Diskussion, wenn die Vorschläge zur Regulierung als wissenschaftsfeindlich, religiös motiviert oder den Wissenschaftsstandort gefährdend abgetan werden, wird gerade nicht geführt. Und in Deutschland? Dass es vor allem Vertreter der Akademie der Wissenschaften sowie des Deutschen Ethikrats sind, die sich einmal mehr für eine Kehrtwende in der deutschen Forschungspolitik und für die Liberalisierung des Embryonenschutzgesetzes aussprechen, ist nicht verwunderlich [7] - sie folgen damit nur ihren Kollegen und Kolleginnen aus den Ländern, die immer schon lieber unverbindliche Richtlinien als Gesetze haben wollten und die schon immer die ethischen «Bedenkenträger» als forschungshemmend und die Öffentlichkeit als nicht genügend informiert desavouiert haben.

Wenn aber die derzeitigen Stellungnahmen eine sehr einseitige Geschichte erzählen, eine Geschichte nämlich, in der uns die Technik und die Zukunft mit den neuen Möglichkeiten der genetischen Interventionen schöngeredet wird, ohne die sozialen und wissenschaftspolitischen Konstellationen zu berücksichtigen dann werden es die zukünftigen Generationen sein, die den Preis für unsere mangelnde kritische Urteilskraft zahlen müssen.

Interessenkonflikt: Die Autorin bestätigt, dass hinsichtlich dieses Beitrags keine Interessenkonflikte bestehen.

\section{Abstract \\ The genetic modification of embryos imposes an unreasonable burden on children}

Research on human embryos is subject to special scrutiny in most countries, which is also reflected in international instruments dealing with biomedicine. In view of the fact that alterations in the human germline have now become possible by means of the so-called "gene editing" or "genome editing”, a public discussion on this matter is urgently needed, and maybe even overdue. Ethics must play a greater role in these discussions than the one it is currently playing. The ethical considerations in this article exclusively concern the recourse to gene editing for reproductive purposes. There are, however, important ethical issues to discuss concerning other applications of the technique, such as embryo research per se, embryonic stem cell research, gene editing in somatic gene therapy (especially in the area of gene alterations in fetuses and incapacitated 
persons), the relationship between somatic gene therapy and germline interventions and, not least, the application of gene editing for non-therapeutic purposes, such as enhancement.

\section{Résumé}

\section{La modification génétique des embryons impose aux enfants une contrainte déraisonnable}

La recherche sur l'embryon humain est dans la plupart des pays soumise à des restrictions particulières, ce qui se reflète aussi dans les instruments internationaux sur la biomédecine. Compte tenu des récentes découvertes qui permettent d'introduire des modifications dans la lignée germinale humaine au moyen de l'ingénierie du génome, il semble bien que la discussion éthique est urgente, si elle n'arrive pas déjà en retard. Dans ce domaine, l'éthique devrait jouer un rôle plus important que celui qu'elle joue actuellement. Les réflexions dans cet article portent exclusivement sur l'application de l'ingénierie du génome dans le champ de la reproduction. Cependant, il y a bien d'autres domaines d'application de cette méthode qui suscitent des questionnements éthiques: les recherches sur l'embryon et sur les cellules souches embryonnaires, la thérapie génique somatique lorsqu'elle implique des modifications chez les fœtus et les personnes incapables de donner leur consentement, le rapport entre la thérapie génique somatique et les interventions dans la lignée germinale à des fins thérapeutiques et l'application des méthodes d'ingénierie du génome à des fins non thérapeutiques, par exemple, à des fins d'amélioration.

\section{Korrespondenz}

Professor Hille Haker

Richard McCormick Chair of MoralTheology

Loyola University Chicago

College of Arts and Sciences, CC 321

1032 W. Sheridan Rd.

US-Chicago, 60660

E-Mail: hhaker[at]luc.edu

Eingang des Manuskripts: 3.5.2017

Eingang des überarbeiteten Manuskripts: 25.5.2017

Annahme des Manuskripts: 2.6.2017

\section{Referenzen}

1. Universal Declaration on the Human Genome and Human Rights, 1997.

2. UNESCO International Bioethics Committee. Report of the IBC on Updating Its Reflection on the Human Genome and Human Rights. New York: UNESCO; 2015. Abrufbar unter: http://unesdoc.unesco. org/images/0023/002332/233258E.pdf (Zugriff: 2.5.2017).

3. Council of Europe. Convention for the Protection of Human Rights and Dignity of the Human Being with regard to the Application of Biology and Medicine: Convention on Human Rights and Biomedicine, 1997.

4. Araki M, Ishii T. International regulatory landscape and integration of corrective genome editing into in vitro fertilization. Reproductive Biology and Endocrinology. 2014;12:108.

5. UK Nuffield Council on Bioethics. Genome editing. An ethical review. September 2016. Abrufbar unter: http://nuffieldbioethics.org/ wp-content/uploads/Genome-editing-an-ethical-review.pdf (Zugriff: 2.5.2017).

6. Haker H. Societal Implications of Gene Editing (Presentation). National Academy of Science, Summit Meeting on Human Gene Editing. 1 December 2015. Abrufbar unter:

www.nationalacademies.org/gene-editing/Gene-Edit-Summit/ Slide-Presentations/index.htm (Zugriff: 2.5.2017).

7. Organizing Committee for the International Summit on Human Gene Editing. International Summit Statement. Washington, DC, 3 December 2015. Abrufbar unter: www8.nationalacademies.org/ onpinews/newsitem.aspx?RecordID=12032015a (Zugriff: 2.5.2017).

8. National Academy of Science \& National Academy of Medicine. Human Genome Editing: Science, Ethics, and Governance. 2016. Abrufbar unter: http://nationalacademies.org/gene-editing/ consensus-study/ (Zugriff: 2.5.2017).

9. Harris J. Why human gene editing must not be stopped. The Guardian, Dec 2, 2015. Abrufbar unter: www.theguardian.com/ science/2015/dec/02/why-human-gene-editing-must-not-bestopped (Zugriff: 2.5.2017).

10. Hurley MN, McKeever TM, et al. Rate of improvement of CF life expectancy exceeds that of general population - Observational death registration study. Journal of Cystic Fibrosis. 2014;13:410-5.

11. Harness-Brumley CL, Elliott AC, Rosenbluth DB, et al. Gender differences in outcomes of patients with cystic fibrosis. Journal of women's health. 2014;23(12):1012-20.

12. Haker H. Reproductive rights and reproductive technologies. In: Widdows H, Moellendorf D (eds). The Routledge Handbook of Global Ethics. London: Routledge; 2014, p. 340-53.

13. Haker H. Ethik der genetischen Frühdiagnostik. Sozialethische Reflexionen zur Verantwortung am menschlichen Lebensbeginn. Paderborn: Mentis; 2002.

14. Haker H. Hauptsache gesund? Ethische Fragen der Pränatal- und Präimplantationsdiagnostik. München: Kösel; 2011. 